\title{
Neutral bleiben
}

\author{
WISSENSCHAFT ERKLÄRT: OBJEKTIVITÄT Objektivität ist ursprünglich ein Begriff \\ aus der Naturwissenschaft. Warum dieses Prinzip auch für Ergotherapeuten bei der \\ Anwendung von Assessments gilt, erklärt Jan Mehrholz.
}

\section{0}

bjektiv ist das Gegenteil von subjektiv.

Wenn eine Ergotherapeutin beispielsweise die Bewegungsqualität eines Klienten oder die Arbeit einer Kollegin einschätzt, dann ist das subjektiv. Objektiv dagegen ist die in der Elektro-Myographie (EMG) gemessene elektrische Aktivität eines Muskels oder die Gesamtzahl unentschuldigter Fehlstunden im Pädiatrieunterricht.

Vom edlen Ansatz zum Mythos > Sowohl bei Tests als auch in Studien sollte das Ziel sein, möglichst objektiv und nicht subjektiv zu beurteilen. Die klassische Testtheorie bezeichnet Objektivität als ein Gütekriterium von Messinstrumenten. Damit ist gemeint, dass keinerlei subjektiv gefärbte Interpretationen das gemessene Ergebnis beeinflussen. Die Versuchsergebnisse sollten unabhängig von räumlichen Bedingungen und dem Untersucher sein [1]. Ein Bewegungsanalysesystem zum Beispiel sollte prinzipiell Alltagsbewegungen erfassen - unabhängig von der Klinik, dem Heim, der Wohnung und der Ergotherapeutin. Ein edler Ansatz, aber leider ist absolute Objektivität ein Mythos!

Viele Forscher kritisieren die betont objektive Vorgehensweise von Naturwissenschaftlern, weil jene viele Fraggestellungen aus der Bewegungswissenschaft nur ungenügend beantworten [2]. Selbst objektiv erhobene Messungen mit Instrumenten, die fast unverfälscht messen können, werden durch den Versuchsaufbau und die Versuchsanordnung, also den Untersucher, sowie die Ziele und die Interpretation des Untersuchers potenziell beeinflusst [3]. Angefangen von der Untersuchungsidee bis hin zur Auswahl des Messinstruments oder Assessmentverfahrens ist der Untersuchende beteiligt und nicht nur außenstehend [1].
Schritt 1: Durchführung > Das Gütekriterium Objektivität gilt für drei Abschnitte einer Untersuchung: Durchführung, Auswertung und Interpretation. Mit der Durchführungsobjektivität ist die Unabhängigkeit der Testergebnisse vom Versuchsleiter gemeint. Je besser Tests standardisiert sind und die zu untersuchende Aktivität klar definieren, wie beispielsweise die Alltagsaktivität „Aufstehen“, und je weniger der Untersucher mit dem zu Testenden kommuniziert oder interagiert, umso besser und umso objektiver. Der sogenannte Rosenthal-Effekt beschreibt diese Beeinflussung von Ergebnissen durch die Erwartungen des Untersuchers. Ergotherapeuten sollten demnach Tests aller Art so ergebnisoffen wie möglich durchführen und beispielsweise keine Suggestivfragen stellen.

Schritt 2: Auswertung > Mit Auswertungsobjektivität ist gemeint, dass die Untersucher Messergebnisse nach Möglichkeit immer in der gleichen Weise und möglichst ergebnisneutral auswerten sollten. Mehrere Ergotherapeuten sollten mit dem gleichen Test immer zum gleichen Ergebnis kommen. Wenn ein Forscher bei der Auswertung einer Vergleichsstudie beispielsweise eine Gruppe von Klienten bewusst oder unbewusst bevorzugt, führt dies zu Verzerrungen der Ergebnisse und somit zum Gegenteil von Objektivität. Forscher können die Auswertungsobjektivität in kontrollierten Studien deutlich erhöhen, indem sie zum Beispiel standardisierte Videoaufnahmen nutzen und sogenannte verblindete Auswerter einsetzen, die nicht wissen, welcher Gruppe die Klienten angehören.

Schritt 3: Interpretation > Interpretationsobjektivität schließlich bedeutet, dass Forscher die Interpretation von Testergebnissen

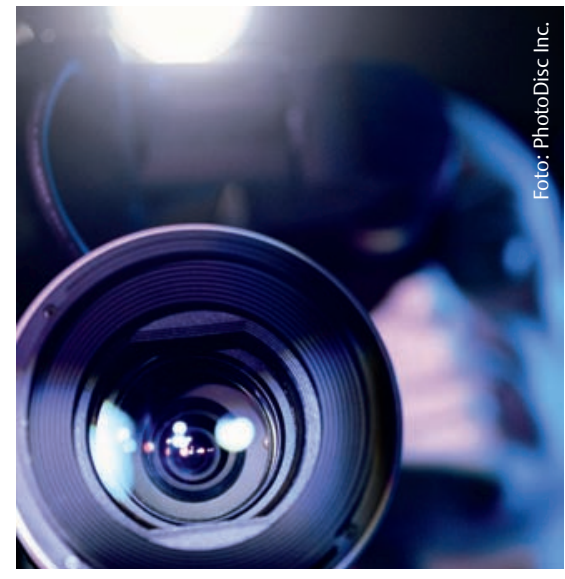

Forscher sollten möglichst objektiv auf ihre Untersuchungen blicken.

und die klinische Relevanz eines Therapieeffekts zuvor festlegen und standardisieren sollten, um diese nicht bewusst oder unbewusst (fehl)zuinterpretieren. Ein Testergebnis sollte durch verschiedene Ergotherapeuten gleich interpretierbar sein. Ein Beispiel für schlechte Interpretationsobjektivität ist das Konstrukt „Tonus“, das verschiedene Therapeuten verschieden auslegen können. Ein positives Beispiel hingegen ist die Einschätzung der Wachheit eines Klienten anhand der Koma-Remissions-Skala.

Jan Mehrholz

$\Rightarrow$ Das Literaturverzeichnis finden Sie unter www.thieme.de/ergoonline > ,ergopraxis“ > "Artikel“ > „Wissenschaft erklärt“.

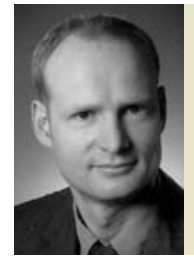

Prof. Dr. Jan Mehrholz, Leiter des Wissenschaftlichen Instituts der Privaten Europäischen Medizinischen Akademie in Kreischa und in Gera Professor für Therapiewissenschaften 